\title{
Weighted sums of orthogonal polynomials with positive zeros
}

\author{
Masaaki Kijima ${ }^{a}$, Erik A. van Doorn ${ }^{b, *}$ \\ ${ }^{a}$ Graduate School of Systems Management, University of Tsukuba, Tokyo, Japan \\ ${ }^{\mathrm{b}}$ Faculty of Applied Mathematics, University of Twente, P.O. Box 217, 7500 AE Enschede, Netherlands
}

Received 4 November 1994; revised 8 February 1995

\begin{abstract}
We study the two sequences of polynomials which arise as denominators of the approximants of even and odd order, respectively, of a Stieltjes fraction, and which may be defined alternatively as a sequence of orthogonal polynomials with positive zeros and the associated sequence of kernel polynomials. Motivated by problems in the setting of birth-death processes, where these sequences play a major role, we focus on the asymptotic behaviour of the sequences and establish convergence of certain weighted sums of the polynomials at hand.
\end{abstract}

Keywords: Stieltjes fraction; Orthogonal polynomials; Birth-death processes; Weighted sums

AMS Classification: $42 \mathrm{C} 05 ; 60 \mathrm{~J} 80$

\section{Introduction}

The polynomials $R_{n}(x)$ defined by the recurrence relation

$$
\begin{aligned}
& \gamma_{2 n+2} R_{n+1}(x)=\left(\gamma_{2 n+1}+\gamma_{2 n+2}-x\right) R_{n}(x)-\gamma_{2 n+1} R_{n-1}(x), \quad n \geqslant 1, \\
& R_{0}(x)=1, \quad \gamma_{2} R_{1}(x)=\gamma_{2}-x,
\end{aligned}
$$

where $\gamma_{n}>0$, arise in Stieltjes' famous memoir [9] as denominators of the approximants of even order of the continued fraction

$$
\frac{1}{\mid a_{1} z}+\frac{1}{\mid a_{2}}+\frac{1}{\mid a_{3} z}+\frac{1}{\mid a_{4}}+\cdots,
$$

if we let $x=-z$ and $\gamma_{n+1}=\left(a_{n} a_{n+1}\right)^{-1}$. Evidently, by Favard's theorem, the sequence $\left\{R_{n}(x)\right\}_{n=0}^{\infty}$ constitutes an orthogonal polynomial sequence (with respect to a positive-definite moment

*Corresponding author. E-mail: doorn@math.utwente.nl.

0377-0427/95/\$09.50 (C) 1995 Elsevier Science B.V. All rights reserved

SSDI 0377-0427(95)00105-0 
functional). In addition, Chihara [2] (see also the Corollary to [4, Theorem I.9.1]) has observed that the existence of positive numbers $\gamma_{n}, n \geqslant 2$, satisfying (1.1) is necessary and sufficient for a sequence of orthogonal polynomials $\left\{R_{n}(x)\right\}$ to be orthogonal on $[0, \infty)$, that is, to have only positive zeros.

The sequence $\left\{R_{n}(x)\right\}$ plays an important role also in the analysis of birth-death processes on the nonnegative integers for which state 0 is a reflecting barrier, if we interpret $\gamma_{2 n+1}$ as the death rate and $\gamma_{2 n+2}$ as the birth rate in state $n$. For example, the transition probabilities of such a birth-death process can be represented in terms of the polynomials $R_{n}(x)$ and their orthogonalizing measure, see [6].

Associated with $\left\{R_{n}(x)\right\}$ is the sequence $\left\{R_{n}^{*}(x)\right\}$ of polynomials satisfying the recurrence

$$
\begin{aligned}
& \gamma_{2 n+3} R_{n+1}^{*}(x)=\left(\gamma_{2 n+2}+\gamma_{2 n+3}-x\right) R_{n}^{*}(x)-\gamma_{2 n+2} R_{n-1}^{*}(x), \quad n \geqslant 1, \\
& R_{0}^{*}(x)=1, \quad \gamma_{3} R_{1}^{*}(x)=\gamma_{2}+\gamma_{3}-x,
\end{aligned}
$$

which are related to the odd-order approximants of the continued fraction (1.2). Again we are dealing with a sequence of orthogonal polynomials whose zeros are positive. In the context of birth-death processes with an absorbing state -1 (and a positive death rate in state 0 ) the polynomials $R_{n}^{*}(x)$ play a role similar to that of $R_{n}(x)$ for birth-death processes with a reflecting state 0 , if we interpret $\gamma_{2 n+2}$ as the death rate and $\gamma_{2 n+3}$ as the birth rate in state $n$, see [6].

Motivated by problems in the setting of birth-death processes we study convergence of the series

$$
\sum_{n=0}^{\infty} c_{n} R_{n}(x) \text { and } \sum_{n=0}^{\infty} c_{n}^{*} R_{n}^{*}(x)
$$

for $x \geqslant 0$ and certain constants $c_{n}$ and $c_{n}^{*}$ depending on the parameters $\left\{\gamma_{n}\right\}$. In particular we want to establish under which conditions certain series of the type

$$
x \sum_{n=0}^{\infty} c_{n} R_{n}(x) \text { and } x \sum_{n=0}^{\infty} c_{n}^{*} R_{n}^{*}(x)
$$

sum up to 1 , so that the sequences $\left\{c_{n} x R_{n}(x)\right\}_{n=0}^{\infty}$ and $\left\{c_{n}^{*} x R_{n}^{*}(x)\right\}_{n=0}^{\infty}$ represent probability distributions on the nonnegative integers when their elements are nonnegative.

The constants $c_{n}$ and $c_{n}^{*}$ in (1.4) arise from probabilistic considerations, but also quite naturally from studying convergence of the sequences $\left\{R_{n}(x)\right\}$ and $\left\{R_{n}^{*}(x)\right\}$. Actually, it is the latter approach we choose in this paper. Thus, after having established a number of preliminary results in Section 2 , we set forth the asymptotic behaviour as $n \rightarrow \infty$ of the sequences $\left\{R_{n}(x)\right\}$ and $\left\{R_{n}^{*}(x)\right\}$ in Section 3 . Then, in Section 4, we show by exploiting a number of identities relating the sequences $\left\{R_{n}(x)\right\}$ and $\left\{R_{n}^{*}(x)\right\}$, how the asymptotic results of Section 3 lead to statements about weighted sums of the type (1.4).

Our findings may be viewed as extensions of the work on the asymptotic behaviour of the polynomial sequences $\left\{R_{n}(x)\right\}$ and $\left\{R_{n}^{*}(x)\right\}$ already begun by Stieltjes in [9] and continued by others in $[3,6,11]$. The probabilistic implications of our results are elaborated elsewhere [7]. 


\section{Preliminaries}

We find it convenient to commence our analysis by considering the sequence $\mathscr{P} \equiv\left\{P_{n}(x)\right\}_{n=0}^{\infty}$ of monic polynomials satisfying the recurrence relation

$$
\begin{aligned}
& P_{n}(x)=\left(x-\gamma_{2 n-1}-\gamma_{2 n}\right) P_{n-1}(x)-\gamma_{2 n-2} \gamma_{2 n-1} P_{n-2}(x), \quad n \geqslant 2, \\
& P_{0}(x)=1, \quad P_{1}(x)=x-\gamma_{2},
\end{aligned}
$$

where $\gamma_{n}, n \geqslant 2$, are the positive parameters of (1.1). It is easily seen that

$$
P_{n}(0)=(-1)^{n} \prod_{i=1}^{n} \gamma_{2 i}, \quad n \geqslant 0,
$$

where we use the convention (which is maintained throughout this paper) that an empty product denotes unity. The polynomials $R_{n}(x)$ of (1.1) can now be expressed as

$$
R_{n}(x)=P_{n}(x) / P_{n}(0), \quad n \geqslant 0 .
$$

By $\mathscr{P}^{*} \equiv\left\{P_{n}^{*}(x)\right\}_{n=0}^{\infty}$ we denote the sequence of kernel polynomials (with parameter 0 ) associated with $\mathscr{P}$, that is

$$
\begin{aligned}
x P_{n}^{*}(x) & =P_{n+1}(x)-\left(P_{n+1}(0) / P_{n}(0)\right) P_{n}(x) \\
& =P_{n+1}(x)+\gamma_{2 n+2} P_{n}(x), \quad n \geqslant 0,
\end{aligned}
$$

see [4]. These kernel polynomials then satisfy the recurrence relation

$$
\begin{aligned}
& P_{n}^{*}(x)=\left(x-\gamma_{2 n}-\gamma_{2 n+1}\right) P_{n-1}^{*}(x)-\gamma_{2 n-1} \gamma_{2 n} P_{n-2}^{*}, \quad n \geqslant 2, \\
& P_{0}^{*}(x)=1, \quad P_{1}^{*}(x)=x-\gamma_{2}-\gamma_{3} .
\end{aligned}
$$

With the notation

$$
G_{n} \equiv \prod_{i=1}^{n}\left(\gamma_{2 i} / \gamma_{2 i+1}\right), \quad K_{n} \equiv \sum_{i=0}^{n} G_{i}, \quad n \geqslant 0,
$$

it is not difficult to verify that

$$
P_{n}^{*}(0)=(-1)^{n} K_{n} \prod_{i=1}^{n} \gamma_{2 i+1}, \quad n \geqslant 0 .
$$

It follows that the polynomials $R_{n}^{*}(x)$ of $(1.3)$ can be represented as

$$
R_{n}^{*}(x)=K_{n} P_{n}^{*}(x) / P_{n}^{*}(0), \quad n \geqslant 0 .
$$

Remark 2.1. Clearly, the recurrence relations (2.1) for $\mathscr{P}$ and (2.5) for $\mathscr{P}^{*}$ are structurally different. However, defining $\gamma_{2 n}^{*} \equiv \gamma_{2 n+1} K_{n} / K_{n-1}$ and $\gamma_{2 n+1}^{*} \equiv \gamma_{2 n+2} K_{n-1} / K_{n}$, it is not difficult to see 
that the sequence $\mathscr{P}^{*}$ satisfies the recurrence (2.1) with $\gamma_{n}$ replaced by $\gamma_{n}^{*}$. Hence, with appropriate interpretation of the parameters involved, any result for $\mathscr{P}$ is valid for $\mathscr{P}^{*}$ as well.

Since the sequences $\mathscr{P}$ and $\mathscr{P}^{*}$ constitute orthogonal polynomial sequences, $P_{n}(x)$ and $P_{n}^{*}(x)$ have $n$ real, simple zeros $x_{n 1}<x_{n 2}<\cdots<x_{n n}$ and $x_{n 1}^{*}<x_{n 2}^{*}<\cdots<x_{n n}^{*}$, respectively, which satisfy the separation properties

$$
x_{n+1, i}<x_{n i}<x_{n+1, i+1}, \quad i=1,2, \ldots, n, n \geqslant 1,
$$

and

$$
x_{n+1, i}^{*}<x_{n i}^{*}<x_{n+1, i+1}^{*}, \quad i=1,2, \ldots, n, n \geqslant 1 .
$$

We have mentioned in Section 1 that all zeros of the polynomials $R_{n}(x)$, and hence $P_{n}(x)$, are positive. Combining this result with the separation property in [4, Theorem I.7.2] gives us

$$
0<x_{n i}<x_{n i}^{*}<x_{n+1, i+1}, \quad i=1,2, \ldots, n, n \geqslant 1 .
$$

It follows from (2.9) and (2.10) that the limits

$$
\zeta_{i} \equiv \lim _{n \rightarrow \infty} x_{n i} \quad \text { and } \quad \zeta_{i}^{*} \equiv \lim _{n \rightarrow \infty} x_{n i}^{*}, \quad i \geqslant 1,
$$

exist, and by (2.11) we have

$$
0 \leqslant \zeta_{i} \leqslant \zeta_{i}^{*} \leqslant \zeta_{i+1}<\infty, \quad i \geqslant 1
$$

Subsequently defining

$$
\sigma \equiv \lim _{i \rightarrow \infty} \zeta_{i} \text { and } \sigma^{*} \equiv \lim _{i \rightarrow \infty} \zeta_{i}^{*},
$$

we conclude $0 \leqslant \sigma \leqslant \sigma^{*} \leqslant \infty$. It is also interesting to note that, for $i \geqslant 1$,

$$
\zeta_{i}=\zeta_{i+1} \quad \Rightarrow \quad \zeta_{i}=\sigma,
$$

see [4, Theorem II.4.6]. The analogue of (2.13) for $\mathscr{P}^{*}$ is obviously valid as well, cf. Remark 2.1 .

The quantities $\zeta_{i}$ and $\zeta_{i}^{*}$ have a prominent part in what follows, and our first task will be to obtain results which are more precise than (2.12). Besides the quantities $G_{n}$ and $K_{n}$ introduced in (2.6) we shall use

$$
H_{n} \equiv \gamma_{2}^{-1} \prod_{i=1}^{n}\left(\gamma_{2 i+1} / \gamma_{2 i+2}\right), \quad L_{n} \equiv \sum_{i=0}^{n} H_{i}, \quad n \geqslant 0,
$$

where we follow [11] and deviate slightly from the notation in [5]. In line with (2.6) and (2.14) we let

$$
K_{\infty} \equiv \sum_{n=0}^{\infty} G_{n}, \quad L_{\infty} \equiv \sum_{n=0}^{\infty} H_{n}
$$


and note that

$$
\sum_{n=0}^{\infty} G_{n+1} L_{n}+\sum_{n=0}^{\infty} H_{n} K_{n}<\infty \quad \Longleftrightarrow K_{\infty}+L_{\infty}<\infty,
$$

as can be easily verified. In the next theorem $\zeta^{-1}$ should be interpreted as infinity if $\zeta=0$.

Theorem 2.2. (i) If $K_{\infty}+L_{\infty}<\infty$, then $\sum_{i=1}^{\infty} \zeta_{i}^{-1}<\infty$ and

$$
0<\zeta_{i}<\zeta_{i}^{*}<\zeta_{i+1}, \quad i \geqslant 1 \text {. }
$$

(ii) If $\sum_{n} G_{n+1} L_{n}=\infty$ and $\sum_{n} H_{n} K_{n}<\infty$, then $\sum_{i=1}^{\infty} \zeta_{i}^{-1}<\infty$ and

$$
0<\zeta_{i}=\zeta_{i}^{*}<\zeta_{i+1}, \quad i \geqslant 1 .
$$

(iii) If $\sum_{n} G_{n+1} L_{n}<\infty$ and $\sum_{n} H_{n} K_{n}=\infty$, then $\sum_{i=2}^{\infty} \zeta_{i}^{-1}<\infty$ and

$$
0=\zeta_{1}<\zeta_{i}^{*}=\zeta_{t+1}<\zeta_{i+1}^{*}, \quad i \geqslant 1 \text {. }
$$

(iv) If $\sum_{n} G_{n+1} L_{n}=\sum_{n} H_{n} K_{n}=\infty$, then $\sum_{i=2}^{\infty} \zeta_{i}^{-1}=\infty$ and either

$$
\zeta_{1}=0 \quad \text { and } \quad \zeta_{i}^{*}=\zeta_{i+1}, \quad i \geqslant 1,
$$

or

$$
\zeta_{1}>0 \text { and } \zeta_{i}^{*}=\zeta_{i}, \quad i \geqslant 1 .
$$

Moreover,

$$
\begin{aligned}
& K_{\infty}<\infty \quad \Rightarrow \quad \zeta_{1}=0, \\
& K_{\infty}=L_{\infty}=\infty \quad \Rightarrow \quad \sigma=0
\end{aligned}
$$

and

$$
K_{\infty}=\infty \quad \text { and } \quad \zeta_{1}=0 \Rightarrow \sigma=0 .
$$

Proof. Part (i) can be found in [5, p. 340], while the last three results of part (iv) follow from Theorem 4 of the same paper. The other results are implied by Theorems 1, 2 and 3 in [11].

Remark 2.3. Note that $\sigma \equiv \lim _{i \rightarrow \infty} \zeta_{i}=\infty$ if $\sum_{i=2}^{\infty} \zeta_{i}^{-1}<\infty$; the reverse does not necessarily hold true.

Our next preparatory task is to obtain some identities involving the polynomials $\left\{R_{n}(x)\right\}$ and $\left\{R_{n}^{*}(x)\right\}$. First, in view of (2.3) and (2.8), relation (3.4) in [11] may be written as

$$
R_{n}^{*}(x)=\sum_{i=0}^{n} G_{i} R_{i}(x), \quad n \geqslant 0 .
$$


Combining this result with $(2.2),(2.4)$ and (2.7) readily yields

$$
R_{n+1}(x)=1-x \sum_{i=0}^{n} H_{i} R_{i}^{*}(x), \quad n \geqslant 0 .
$$

Substitution of (2.17) into (2.18) subsequently gives us

$$
R_{n+1}(x)=1-x \sum_{i=0}^{n} H_{i} \sum_{j=0}^{i} G_{j} R_{j}(x), \quad n \geqslant 0,
$$

which may be written as

$$
L_{n}^{-1} R_{n+1}(x)=L_{n}^{-1}-x \sum_{i=0}^{n} G_{i}\left(1-L_{n}^{-1} L_{i-1}\right) R_{i}(x), \quad n \geqslant 0,
$$

where $L_{-1} \equiv 0$. On the other hand, substitution of (2.18) into (2.17) gives us

$$
R_{n+1}^{*}(x)=K_{n+1}-x \sum_{i=0}^{n} G_{i+1} \sum_{j=0}^{i} H_{j} R_{j}^{*}(x), \quad n \geqslant 0,
$$

which may be written as

$$
K_{n+1}^{-1} R_{n+1}^{*}(x)=1-x \sum_{i=0}^{n} H_{i}\left(1-K_{n+1}^{-1} K_{i}\right) R_{i}^{*}(x), \quad n \geqslant 0 .
$$

Our final group of preparatory results concern monotonic behaviour of the sequences $\left\{R_{n}(x)\right\}$ and $\left\{R_{n}^{*}(x)\right\}$. Our principal tool to establish results of this type is the Basic Oscillation Theorem in [10], which, when applied to $\mathscr{P}$ and $\mathscr{P}^{*}$, respectively, tells us that for any $k \geqslant 0$

$$
\mathscr{S}\left\{R_{n}(x)\right\}=k \quad \Longleftrightarrow \quad \zeta_{k}<x \leqslant \zeta_{k+1}
$$

and

$$
\mathscr{S}\left\{R_{n}^{*}(x)\right\}=k \quad \Longleftrightarrow \quad \zeta_{k}^{*}<x \leqslant \zeta_{k+1}^{*} .
$$

Here $\zeta_{0}=\zeta_{0}^{*} \equiv-\infty$, and, for any sequence $\left\{a_{n}\right\}_{n=0}^{\infty}, \mathscr{S}\left\{a_{n}\right\}$ denotes the number of sign changes in the sequence $\left\{a_{n}\right\}$ after deleting all zero elements. (By convention, $\mathscr{S}\left\{a_{n}\right\} \equiv-1$ if $a_{n}=0$ for all $n$.) Indeed, by combining (2.17), (2.18), (2.23), (2.24) and Theorem 2.2, various monotonicity results are easily brought to light. In the next three lemmas we have gathered those results for which we have use in the next section.

Lemma 2.4. If $x<0$, then both $\left\{R_{n}(x)\right\}_{n=0}^{\infty}$ and $\left\{R_{n}^{*}(x)\right\}_{n=0}^{\infty}$ are positive and increasing.

Lemma 2.5. Let $\zeta_{1}>0$ and $K_{\infty}+L_{\infty}=\infty$.

(i) If $0<x \leqslant \zeta_{1}$, then $\left\{R_{n}(x)\right\}_{n=0}^{\infty}$ is positive and decreasing, while $\left\{R_{n}^{*}(x)\right\}_{n=0}^{\infty}$ is positive and increasing. 
(ii) If $\zeta_{k}<x \leqslant \zeta_{k+1}$ for some $k \geqslant 1$, then $\left\{(-1)^{k} R_{n}(x)\right\}_{n=N}^{\infty}$ is positive and decreasing, while $\left\{(-1)^{k} R_{n}^{*}(x)\right\}_{n=N}^{\infty}$ is positive and increasing, for $N$ sufficiently large.

Lemma 2.6. Let $\zeta_{1}=0$ (so that $K_{\infty}+L_{\infty}=\infty$ ). If $\zeta_{k}<x \leqslant \zeta_{k+1}$ for some $k \geqslant 1$, then $\left\{(-1)^{k} R_{n}(x)\right\}_{n=N}^{\infty}$ is positive and increasing for $N$ sufficiently large (increasing for $N=0$ if $k=1$ ), while $\left\{(-1)^{k} R_{n}^{*}(x)\right\}_{n=N}^{\infty}$ is negative and increasing for $N$ sufficiently large (negative for $N=0$ if $k=1)$.

These lemmas are extensions of Lemmas 3 and 4 in [11].

We have now gathered sufficient information to commence our analysis of the limiting behaviour of the polynomial sequences $\left\{R_{n}(x)\right\}$ and $\left\{R_{n}^{*}(x)\right\}$ in the next section.

\section{Limits}

Our first result in this section is a restatement of Theorem 1 in [11], parts of which can be found also in $[3,6,9]$.

Theorem 3.1. The following statements are equivalent:

(i) $\sum_{i=1}^{\infty} \zeta_{i}^{-1}<\infty$,

(ii) $\sum_{n=0}^{\infty} H_{n} K_{n}<\infty$,

(iii) $\left\{R_{n}(x)\right\}_{n}$ converges uniformly on bounded sets to an entire function whose zeros are simple and are precisely the points $\zeta_{i}, i \geqslant 1$,

(iv) $\left\{R_{n}(x)\right\}_{n}$ is bounded as $n \rightarrow \infty$ for at least one $x<0$.

In view of Remark 2.1 we can interpret this theorem in terms of $\mathscr{P}^{*}$, which after some algebra gives the next corollary.

Corollary 3.2. The following statements are equivalent:

(i) $\sum_{i=1}^{\infty}\left(\zeta_{i}^{*}\right)^{-1}<\infty$,

(ii) $\sum_{n=0}^{\infty} G_{n+1} L_{n}<\infty$ or $\sum_{n=0}^{\infty} H_{n} K_{n}<\infty$,

(iii) $\left\{P_{n}^{*}(x) / P_{n}^{*}(0)\right\}_{n}$ converges uniformly on bounded sets to an entire function whose zeros are simple and are precisely the points $\zeta_{i}^{*}, i \geqslant 1$,

(iv) $\left\{P_{n}^{*}(x) / P_{n}^{*}(0)\right\}_{n}$ is bounded as $n \rightarrow \infty$ for at least one $x<0$.

This corollary leads to a partial analogue of Theorem 3.1 in terms of the polynomials $\left\{R_{n}^{*}(x)\right\}_{n}$.

Theorem 3.3. The following statements are equivalent:

(i) $\sum_{n=0}^{\infty} G_{n+1} L_{n}<\infty$,

(ii) $\left\{R_{n}^{*}(x)\right\}_{n}$ converges uniformly on bounded sets to an entire function whose zeros are simple and are precisely the points $\zeta_{i}^{*}, i \geqslant 1$,

(iii) $\left\{R_{n}^{*}(x)\right\}_{n}$ is bounded as $n \rightarrow \infty$ for at least one $x<0$.

Proof. The theorem is almost identical to Lemma 4 in [6], where, however, the statement about the zeros in (ii) is missing. But if (i) holds true, then $K_{\infty} \equiv \sum_{n=0}^{\infty} G_{n}<1+\gamma_{2} \sum_{n=0}^{\infty} G_{n+1} L_{n}<\infty$. So the statement follows by Corollary 3.2 and (2.8). 
We note that the analogy between Theorems 3.1 and 3.3 is not complete, since, by Corollary 3.2, each of the statements in Theorem 3.3 implies $\sum_{i=1}^{\infty}\left(\zeta_{i}^{*}\right)^{-1}<\infty$, but the reverse implications do not necessarily hold true.

From the preceding two theorems we conclude that both $R_{n}(x)$ and $R_{n}^{*}(x)$ tend to entire functions as $n \rightarrow \infty$ if and only if both $\sum_{n} G_{n+1} L_{n}<\infty$ and $\sum_{n} H_{n} K_{n}<\infty$. What happens if precisely one of the series $\sum_{n} G_{n+1} L_{n}$ and $\sum_{n} H_{n} K_{n}$ converges is described in the next theorem, which appears already in Stieltjes' memoir [9]. For the sake of completeness we add a short proof.

Theorem 3.4. (i) If $\sum_{n} G_{n+1} L_{n}=\infty$ and $\sum_{n} H_{n} K_{n}<\infty$, then $\left\{R_{n}(x)\right\}_{n}$ and $\left\{K_{n}^{-1} R_{n}^{*}(x)\right\}_{n}$ tend to the same entire function as $n \rightarrow \infty$.

(ii) If $\sum_{n} G_{n+1} L_{n}<\infty$ and $\sum_{n} H_{n} K_{n}=\infty$, then $\left\{x R_{n}^{*}(x)\right\}_{n}$ and $\left\{-L_{n}^{-1} R_{n+1}(x)\right\}_{n}$ tend to the same entire function as $n \rightarrow \infty$.

Proof. (i) The conditions and (2.16) imply that $K_{n} \equiv \sum_{i=0}^{n} G_{i} \rightarrow K_{\infty}=\infty$ as $n \rightarrow \infty$, while, by (2.17), we have

$$
K_{n}^{-1} R_{n}^{*}(x)=\sum_{i=0}^{n} G_{i} R_{i}(x) / \sum_{i=0}^{n} G_{i} .
$$

The required result follows by Theorem 3.1 and Stolz' criterion, see [1].

(ii) This part is proven similarly with the help of (2.18) and Theorem 3.3.

What remains to be analysed is the case in which both $\sum_{n} G_{n+1} L_{n}$ and $\sum_{n} H_{n} K_{n}$ diverge. If $x<0$, then by Lemma 2.4 both $\left\{R_{n}(x)\right\}_{n}$ and $\left\{R_{n}^{*}(x)\right\}_{n}$ are positive and increasing, while by Theorems 3.1 and 3.3 both are unbounded as $n \rightarrow \infty$. Of course, the case $x=0$ is trivial. So in what follows we restrict our attention to positive values of $x$.

Lemma 3.5. Let $x>0$ and assume $\sum_{n} H_{n} K_{n}=\infty$ and $L_{\infty}<\infty$ (so that $K_{\infty}=\infty$ and hence $\left.\sum_{n} G_{n+1} L_{n}=\infty\right)$. If $R_{n}(x)$ tends to a limit as $n \rightarrow \infty$, then $\lim _{n \rightarrow \infty} R_{n}(x)=0$.

Proof. Suppose $R_{n}(x) \rightarrow a$ as $n \rightarrow \infty$, where $0<a \leqslant \infty$, and let $0<b<a$. Choose $N$ such that $R_{n}(x)>b$ for all $n>N$. Then, for $k$ sufficiently large,

$$
\begin{aligned}
& \sum_{n=0}^{k} H_{n} \sum_{i=0}^{n} G_{i} R_{i}(x) \\
& \quad=\sum_{n=0}^{N} H_{n} \sum_{i=0}^{n} G_{i} R_{i}(x)+\sum_{n=N+1}^{k} H_{n} \sum_{i=0}^{N} G_{i} R_{i}(x)+\sum_{n=N+1}^{k} H_{n} \sum_{i=N+1}^{n} G_{i} R_{i}(x) \\
& \quad>\sum_{n=0}^{N} H_{n} \sum_{i=0}^{n} G_{i} R_{i}(x)+\sum_{n=N+1}^{k} H_{n} \sum_{i=0}^{N} G_{i}\left(R_{i}(x)-b\right)+b \sum_{n=N+1}^{k} H_{n} K_{n} .
\end{aligned}
$$

The assumptions imply that the right-hand side of this inequality tends to infinity as $k \rightarrow \infty$, which, however, contradicts (2.19). Similarly, the supposition $R_{n}(x) \rightarrow a$, with $-\infty \leqslant a<0$, leads to a contradiction. The lemma follows. 
We can now prove one of our main results, which generalizes Theorem 4 in [11] (recall that $\left.\zeta_{0} \equiv-\infty\right)$.

Theorem 3.6. Let $\sum_{n} G_{n+1} L_{n}=\sum_{n} H_{n} K_{n}=\infty, \zeta_{1}>0$ and $\max \left\{0, \zeta_{k}\right\}<x \leqslant \zeta_{k+1}$ for some $k \geqslant 0$. Then $R_{n}(x) \rightarrow 0$ and $(-1)^{k} R_{n}^{*}(x) \rightarrow \infty$ as $n \rightarrow \infty$.

Proof. By Theorem 2.2(iv) we must have $K_{\infty}=\infty$ and $L_{\infty}<\infty$, since $\zeta_{1}>0$. Applying Lemmas 2.5 and 3.5 it follows immediately that $R_{n}(x) \rightarrow 0$ as $n \rightarrow \infty$. Next invoking (2.18) we conclude

$$
(-1)^{k} R_{n}(x)=x \sum_{i=n}^{\infty} H_{i}\left((-1)^{k} R_{i}^{*}(x)\right) .
$$

But Lemma 2.5 tells us that there exists an $N$ such that $\left\{(-1)^{k} R_{i}^{*}(x)\right\}_{i=N}^{\infty}$ is positive and increasing. Consequently,

$$
(-1)^{k} R_{n}(x) \geqslant x r \sum_{i=n}^{\infty} H_{i}, \quad n \geqslant N,
$$

where $r \equiv(-1)^{k} R_{N}^{*}(x)>0$. With (2.17) and (3.2) we now obtain for $n \geqslant N$

$$
\begin{aligned}
(-1)^{k} R_{n}^{*}(x) & =\sum_{i=0}^{N-1} G_{i}\left((-1)^{k} R_{i}(x)\right)+\sum_{i=N}^{n} G_{i}\left((-1)^{k} R_{i}(x)\right) \\
& \geqslant \sum_{i=0}^{N-1} G_{i}\left((-1)^{k} R_{i}(x)\right)+x r \sum_{i=N}^{n} G_{i} \sum_{j=i}^{\infty} H_{j} \\
& =\sum_{i=0}^{N-1} G_{i}\left((-1)^{k} R_{i}(x)\right)-x r \sum_{i=0}^{N-1} G_{i} \sum_{j=i}^{\infty} H_{j}+x r \sum_{i=0}^{n} G_{i} \sum_{j=i}^{\infty} H_{j} \\
& =\sum_{i=0}^{N-1} G_{i}\left((-1)^{k} R_{i}(x)-x r \sum_{j=i}^{\infty} H_{j}\right)+x r \sum_{i=0}^{\infty} H_{i} K_{p},
\end{aligned}
$$

where $p \equiv \min \{i, n\}$. But the right-hand side of this inequality tends to infinity as $n \rightarrow \infty$, since $\sum_{n} H_{n} K_{n}=\infty$. It follows that $(-1)^{k} R_{n}^{*}(x) \rightarrow \infty$ as $n \rightarrow \infty$.

The next lemma and subsequent theorem are the counterparts of Lemma 3.5 and Theorem 3.6, respectively. The theorem generalizes Theorem 5 in [11].

Lemma 3.7. Let $x>0$ and assume $\sum_{n} G_{n+1} L_{n}=\infty$ and $K_{\infty}<\infty$ (so that $L_{\infty}=\infty$ and hence $\left.\sum_{n} H_{n} K_{n}=\infty\right)$. If $R_{n}^{*}(x)$ tends to a limit as $n \rightarrow \infty$, then $\lim _{n \rightarrow \infty} R_{n}^{*}(x)=0$.

Proof. Employing (2.21) instead of (2.19), the proof is completely analogous to that of Lemma 3.5 .

Theorem 3.8. Let $\sum_{n} G_{n+1} L_{n}=\sum_{n} H_{n} K_{n}=\infty, \zeta_{1}=0$ and $\zeta_{k}<x \leqslant \zeta_{k+1}$ for some $k \geqslant 1$. Then $R_{n}^{*}(x) \rightarrow 0$ and $(-1)^{k} R_{n}(x) \rightarrow \infty$ as $n \rightarrow \infty$. 
Proof. Interchanging the roles of (2.17) and (2.18) and using Lemma 2.6 instead of Lemma 2.5, the proof completely parallels that of Theorem 3.6 .

\section{Weighted sums}

In this section we apply the asymptotic results of Theorems $3.1,3.3,3.4,3.6$ and 3.8 to the identities (2.17), (2.18), (2.20) and (2.22) in order to get results on weighted sums of the type (1.4). In what follows we write

$$
R_{\infty}(x) \equiv \lim _{n \rightarrow \infty} R_{n}(x) \quad \text { and } \quad R_{\infty}^{*}(x) \equiv \lim _{n \rightarrow \infty} R_{n}^{*}(x),
$$

if the parameters $\left\{\gamma_{n}\right\}$ are such that the polynomials $R_{n}(x)$ and $R_{n}^{*}(x)$, respectively, tend to entire functions as $n \rightarrow \infty$, see Theorems 3.1 and 3.3.

By combining the results of the latter two theorems with the identities $(2.17),(2.18),(2.20)$ and (2.22) we immediately obtain the next theorem.

Theorem 4.1. (i) If $\sum_{n} G_{n+1} L_{n}<\infty$ (so that $K_{\infty}<\infty$ ), then, for all $x$,

$$
\sum_{n=0}^{\infty} G_{n} R_{n}(x)=R_{\infty}^{*}(x)
$$

and

$$
x \sum_{n=0}^{\infty} H_{n}\left(1-K_{n} / K_{\infty}\right) R_{n}^{*}(x)=1-K_{\infty}^{-1} R_{\infty}^{*}(x) .
$$

(ii) If $\sum_{n} H_{n} K_{n}<\infty$ (so that $L_{\infty}<\infty$ ), then, for all $x$,

$$
x \sum_{n=0}^{\infty} H_{n} R_{n}^{*}(x)=1-R_{\infty}(x)
$$

and

$$
x L_{\infty} \sum_{n=0}^{\infty} G_{n}\left(1-L_{n-1} / L_{\infty}\right) R_{n}(x)=1-R_{\infty}(x) .
$$

For the sake of completeness we also observe the following.

Theorem 4.2. (i) If $\sum_{n} G_{n+1} L_{n}<\infty$ and $\sum_{n} H_{n} K_{n}=\infty$ (so that $K_{\infty}<\infty$ and $L_{\infty}=\infty$ ), then, for $k=0,1, \ldots$,

$$
(-1)^{k+1} x \sum_{n=0}^{\infty} H_{n} R_{n}^{*}(x)=\infty, \quad \zeta_{k}<x<\zeta_{k+1} .
$$

(ii) If $\sum_{n} G_{n+1} L_{n}=\infty$ and $\sum_{n} H_{n} K_{n}<\infty$ (so that $K_{\infty}=\infty$ and $\left.L_{\infty}<\infty\right)$, then, for $k=0,1, \ldots$,

$$
(-1)^{k} \sum_{n=0}^{\infty} G_{n} R_{n}(x)=\infty, \quad \zeta_{k}<x<\zeta_{k+1} .
$$


Proof. (i) From Theorem 3.4(ii) we have $L_{n}^{-1} R_{n+1}(x) \rightarrow-x R_{\infty}^{*}(x)$ as $n \rightarrow \infty$. In view of Theorem 2.2(iii) it follows that for $k=0,1, \ldots$,

$$
(-1)^{k} R_{n}(x) \rightarrow \infty(n \rightarrow \infty), \quad \zeta_{k}<x<\zeta_{k+1} .
$$

The identity (2.18) subsequently yields the required result.

Part (ii) is proven similarly with the help of Theorem 3.4(i), Theorem 2.2(ii) and (2.17).

Note that we have no information on the series in the above theorem if $x=\zeta_{k}$ for some $k \geqslant 1$ (unless $x=\zeta_{1}=0$ in case (i)). We do know by [8, Corollary 2.6], however, that $\sum_{n} H_{n}\left(R_{n}^{*}\left(\zeta_{k}\right)\right)^{2}<$ $\infty$ and $\sum_{n} G_{n}\left(R_{n}\left(\zeta_{k}\right)\right)^{2}<\infty$.

While studying convergence of weighted sums of the types appearing in Theorem 4.1 in the remaining case $\sum_{n} G_{n+1} L_{n}=\sum_{n} H_{n} K_{n}=\infty$, we restrict ourselves to $x \leqslant \sigma$ (if $\sigma=\zeta_{k}$ for some $k$ ) or $x<\sigma$ (if $\sigma>\zeta_{k}$ for all $k$ ). For the other values of $x$ very little can be said in general except that $\left\{R_{n}(x)\right\}_{n}$ and $\left\{R_{n}^{*}(x)\right\}_{n}$ oscillate infinitely often by (2.23) and (2.24). In addition, negative values of $x$ are of little interest since Theorems 3.1 and 3.3, and the identities (2.17), (2.18), (2.20) and (2.22) show that then the pertinent weighted sums are divergent. So, interesting results may arise for $x$ in the interval $[0, \sigma]$, but, in the light of Theorem 2.2(iv), this means that we can confine our attention to the two cases covered by the next theorem.

Theorem 4.3. Let $\sum_{n} G_{n+1} L_{n}=\sum_{n} H_{n} K_{n}=\infty$.

(i) If $K_{\infty}=\infty, L_{\infty}<\infty$ and $\zeta_{1}>0$, then, for $k=0,1, \ldots$,

$$
\begin{aligned}
& x \sum_{n=0}^{\infty} H_{n} R_{n}^{*}(x)=1, \quad 0<x \leqslant \zeta_{k+1}, \\
& (-1)^{k} \sum_{n=0}^{\infty} G_{n} R_{n}(x)=\infty, \quad \max \left\{0, \zeta_{k}\right\}<x \leqslant \zeta_{k+1}, \\
& x L_{\infty} \sum_{n=0}^{\infty} G_{n}\left(1-L_{n-1} / L_{\infty}\right) R_{n}(x)=1, \quad 0<x \leqslant \zeta_{k+1} .
\end{aligned}
$$

(ii) If $K_{\infty}<\infty, L_{\infty}=\infty$ and $\zeta_{1}^{*}>0$, then, for $k=1,2, \ldots$,

$$
\begin{aligned}
& \sum_{n=0}^{\infty} G_{n} R_{n}(x)=0, \quad 0<x \leqslant \zeta_{k+1}, \\
& (-1)^{k-1} \sum_{n=0}^{\infty} H_{n} R_{n}^{*}(x)=\infty, \quad \zeta_{k}<x \leqslant \zeta_{k+1} \\
& \sum_{n=0}^{\infty} H_{n}\left(1-K_{n} / K_{\infty}\right) R_{n}^{*}(x)=1, \quad 0<x \leqslant \zeta_{k+1} .
\end{aligned}
$$

Proof. (i) By (2.12) and Theorem 2.2(iv) we have

$$
0<\zeta_{i}=\zeta_{i}^{*} \leqslant \zeta_{i+1}, \quad i \geqslant 1
$$


Subsequently invoking Theorem 3.6 and, respectively, the identities (2.18) (or (2.22)), (2.17) and (2.20) produce the statements of the first part of the theorem.

(ii) By (2.12) and Theorem 2.2(iv) we now have

$$
0=\zeta_{1}<\zeta_{i}^{*}=\zeta_{i+1} \leqslant \zeta_{i+1}^{*}, \quad i \geqslant 1 .
$$

Hence, Theorem 3.8 and the identities (2.17) (or (2.20)), (2.18) and (2.22), respectively, lead to the statements of the second part.

As mentioned in Section 1 the results of this section are of interest in the analysis of birth-death processes, in particular in the context of limiting conditional distributions, see [7].

\section{Acknowledgements}

We thank Christian Berg for pointing out an omission in an earlier version of this paper.

\section{References}

[1] T.J.I'a Bromwich, An Introduction to the Theory of Infinite Series (MacMillan, London, 2nd ed., 1965).

[2] T.S. Chihara, Chain sequences and orthogonal polynomials, Trans. Amer. Math. Soc. 104 (1962) 1-16.

[3] T.S. Chihara, Convergent sequences of orthogonal polynomials, J. Math. Anal. Appl. 38 (1972) 335-347.

[4] T.S. Chihara, An Introduction to Orthogonal Polynomials (Gordon \& Breach, New York, 1978).

[5] T.S. Chihara, Indeterminate symmetric moment problems, J. Math. Anal. Appl. 85 (1982) 331-346.

[6] S. Karlin and J.L. McGregor, The differential equations of birth-and-death processes, and the Stieltjes moment problem, Trans. Amer. Math. Soc. 85 (1957) 489-546.

[7] M. Kijima, M.G. Nair, P.K. Pollett and E.A. van Doorn, Limiting conditional distributions for birth-death processes, submitted.

[8] J. Shohat and J.D. Tamarkin, The Problem of Moments, Math. Surveys No. 1 (Amer. Math. Soc., Providence, RI, rev. ed., 1963).

[9] T.J. Stieltjes, Recherches sur les fractions continues, Oeuvres Complètes, Vol. 2 (Springer, Berlin, 1994) 406-566.

[10] E.A. van Doorn, On oscillation properties and the interval of orthogonality of orthogonal polynomials, SIAM J. Math. Anal. 15 (1984) 1031-1042.

[11] E.A. van Doorn, On orthogonal polynomials with positive zeros and the associated kernel polynomials, J. Math. Anal. Appl. 113 (1986) 441-450. 